\title{
Usability of liquid crystal displays for research in the temporal characteristics of perception and attention
}

\author{
Ken KiHARA, Jun-ICHIRo KaWAHARA, AND YuJi TAKeda \\ National Institute of Advanced Industrial Science and Technology, Tsukuba, Japan
}

\begin{abstract}
Recently, the use of liquid crystal displays (LCDs) in computer monitors has increased in popularity. Can LCDs produce results similar to those obtained in cathode-ray tube (CRT) displays in studies of temporal attention and perception tasks? Performance in two tasks (metacontrast masking and attentional blink) was examined using an LCD, a CRT oscilloscope, and a raster scan CRT display. Experiment 1 focused on metacontrast masking where a typical metacontrast function emerged irrespective of monitor type. Experiments 2 and 3 examined whether differences in monitors influence the attentional blink. Again, all displays elicited similar performance profiles for both the attentional blink and the trade-off between identification accuracy of the two targets. Although our results may not generalize to all LCD applications and all experimental paradigms, they indicate that LCDs can reproduce results similar to those found in metacontrast masking and attentional blink studies that were originally identified with CRT displays.
\end{abstract}

In order to study the temporal aspects of visual perception and attention, it is necessary to have precise control of the duration of visual stimuli. To this end, computercontrolled monitors are essential. Cathode-ray tube (CRT) oscilloscopes equipped with a very fast fading phosphor, such as $\mathrm{P} 15$, have been considered the best devices in this regard (Di Lollo, Seiffert, Burchett, Rabeeh, \& Ruman, 1997). However, most manufacturers have discontinued these display devices. Color raster-scan CRT displays are also useful because they are easily programmable and provide, in color, relatively fine temporal and spatial resolution of visual images. Thus, until recently, raster scan CRT displays have been a major tool for modern studies of human visual perception (Bach, Meigen, \& Strasburger, 1997; Watson et al., 1986).

Recently, liquid crystal displays (LCDs) have been increasing in popularity for use as computer monitors. LCDs have many advantages over raster scan CRT displays in terms of weight, volume, and electrical power consumption (Menozzi, Näpflin, \& Krueger, 1999). Modern LCDs are also superior to raster scan CRT displays in several aspects of static image quality (Krupinski et al., 2004). Indeed, it has been reported that in terms of assessment of quality, LCDs provide higher quality than do raster scan CRT displays (Tourancheau, Callet, \& Barba, 2008). As prices have fallen, LCDs have been rapidly replacing raster scan CRT displays in homes and offices. Currently, it is difficult to buy a new raster scan CRT display; it appears to be only a matter of time until CRT displays are entirely replaced by LCDs.
A prevalent belief is that LCDs have drawbacks with respect to the ability to control the presentation of dynamic stimuli, when compared with raster scan CRT displays. The temporal characteristics of LCDs depend mainly on the response time required for a pixel to change from one luminance level to another. This response time consists of the liquid crystal response and the modulation of the backlight luminance (Becker, 2008; Elze \& Tanner, 2009). The response time also differs depending on the target gray levels (Elze \& Tanner, 2009; Liang \& Badano, 2007). Wiens et al. (2004) suggested that LCDs (earlier than 2004) were not suitable for psychological experiments requiring high temporal precision in stimulus presentations. They compared the temporal accuracy of an LCD with that of a raster scan CRT display by manipulating the duration of stimulus presentations from 8 to $200 \mathrm{msec}$ and found that the LCD provided poor accuracy: Specifically, the LCD failed to present a target on some trials. However, modern technologies, such as overdrive technology that increases the voltage to force the liquid crystals to boost pixel responsiveness, have improved the temporal performance of LCDs. Such modern LCDs could potentially exhibit comparable performance with raster scan CRT monitors in the temporal domain.

The purpose of the present study was to examine whether behavioral results from perception and attention tasks that have been reported using raster scan CRT displays can be replicated using modern LCDs. As was mentioned previously, a number of studies have reported physical differences in the temporal aspects between

K. Kihara, ken-kihara@aist.go.jp 
LCDs and CRT displays. However, little is known about the differences in psychological or behavioral measures in perception and attention tasks in which visual targets are briefly presented. To address this issue, we compared viewers' identification accuracy of stimuli presented on an LCD with the corresponding accuracy levels for the same stimuli presented on a CRT oscilloscope and on a raster scan CRT display. Specifically, we used two well-known phenomena in which dynamic stimuli are involved: metacontrast masking and attentional blink.

Metacontrast masking is a type of backward masking in which target visibility is reduced when a mask stimulus appears shortly after the target (Alpern, 1953; Breitmeyer \& Öğmen, 2006). In the metacontrast-masking paradigm, the contours of the mask do not overlap spatially with those of the target. The visibility of the target depends on the stimulus onset asynchrony (SOA) between the target and mask. Typically, visibility shows a U-shaped function over SOAs with minimal target identification accuracy around SOAs of 50-100 msec. In this task, the magnitude of metacontrast masking seems to be affected at an early stage of perceptual processing by effects such as spatial proximity of target and mask contours (Breitmeyer \& Öğmen, 2000).

The attentional blink involves a failure to report the second of two targets embedded in a rapid serial visual presentation (RSVP) of distractors with a frequency of about 10/sec (Raymond, Shapiro, \& Arnell, 1992). In general, accuracy of identifying the second target is poor with short SOAs between the two targets, and it gradually increases as the SOA increases. Current theories of the attentional blink suggest that this failure occurs at a relatively late stage of visual processing; specifically, the failure of the second target to be consolidated into working memory is triggered by the processing of the first target (Chun \& Potter, 1995; Shapiro, Arnell, \& Raymond, 1997).

In the present study, we examined whether the effects of metacontrast masking and attentional blink are similar in three types of displays: a modern LCD, a raster scan CRT display, and a CRT oscilloscope. The effects of metacontrast masking and attentional blink were tested in Experiments 1 and 2, respectively.

\section{EXPERIMENT 1}

The purpose of Experiment 1 was to investigate the impact of different display devices on the magnitude of metacontrast masking. Accuracy in target identification was compared using three devices: a CRT oscilloscope, a raster scan CRT display, and an LCD. If differences in temporal characteristics between these devices are critical for an early stage of visual processing, the profile of metacontrast masking should vary across these displays. To be specific, we asked whether the pattern of results obtained with LCDs would deviate substantially from the typical U-shaped function of metacontrast masking.

\section{Method}

Participants. Twelve adults ( 5 of them male and 7 female; age range, 18-27 years) from the participant pool of the National Institute of Advanced Industrial Science and Technology participated in this experiment. All the participants received payment for their participation. All had self-reported normal or corrected-to-normal vision.

Apparatus. The following three sets of monitors were used: a vector scan CRT oscilloscope (Tektronix 608 equipped with P15 phosphor), a raster scan CRT display (Iiyama HF703UG equipped with P22 phosphor), and an LCD (Mitsubishi RDT1714VM; the average response speed was $5 \mathrm{msec}$, according to the manufacturer's specification). Both the raster scan CRT and LCDs were 17 -in. color monitors, set at $1,280 \times 1,024$ pixel resolution, operating at a frame rate of $60 \mathrm{~Hz}$. Although the raster scan CRT display can run at a higher refresh rate $(85 \mathrm{~Hz}$ at maximum), we set it at the same rate as the LCD. A plotting rate of $1000 \mathrm{~Hz}$ was used for the vector scan CRT oscilloscope. Stimuli presented on the raster scan CRT display and the LCD were generated by programs written in MATLAB (The MathWorks, Inc., Natick, MA) with the Psychophysics Toolbox (Brainard, 1997; Pelli, 1997). Stimuli presented on the CRT oscilloscope were controlled by programs written in Turbo C (Borland Inc., Scotts Valley, CA).

Each monitor was turned on at least 30 min before starting experiments in order to settle the brightness of the backlight and the temperature of displays, which affects the response times of the liquid crystal (Liang \& Badano, 2007). The monitors were placed in a dark room, and the viewing distance was $57 \mathrm{~cm}$. Stimuli were presented with green dots in all displays, because the P15 phosphor used in the CRT oscilloscope could present only green stimuli. The maximum luminance of each monitor was adjusted to $50 \mathrm{~cd} / \mathrm{m}^{2}$ by regulating the output signal of the graphics card when a filled green square, subtending $1.2^{\circ}$ of visual angle, was presented continuously in the center of the display. Preceding the experiment, the time course of the luminance change was measured by a calibrated silicon photodiode (S7686, Hamamatsu Photonics) with a neutral density filter. The stimulus presented for measurement involved the onset of the green square, and it lasted $17 \mathrm{msec}$ (i.e., one frame duration of the $60-\mathrm{Hz}$ raster scan) and was followed by $83 \mathrm{msec}$ of blank $\left(<0.3 \mathrm{~cd} / \mathrm{m}^{2}\right)$. The resulting signal, generated by the photodiode, was then amplified by a current-to-voltage conversion amplifier (C9329, Hamamatsu Photonics) and digitized by an analog-digital converter at a sampling rate of $10000 \mathrm{~Hz}$ (DAQCard-6036E, National Instruments). Averages over 100 tests of these time courses, measured as above for each device, are shown in Figure 1. Note that the peak amplitudes of illumination signal in the CRT oscilloscope and the LCD were obviously lower than that in the raster scan CRT display, although all displays were adjusted at $50 \mathrm{~cd} / \mathrm{m}^{2}$ when the stimulus was presented continuously. This is because the luminance signal is integrated over time during the luminance adjustment. To obtain the same area under the luminance curve, the single pulse of the CRT display must be higher than the 17 pulses of the CRT oscilloscope and the continuous signal of the LCD. As well, onsets of illumination signal for the raster scan CRT display and the LCD were delayed from the initial vertical sync signal (approximately $8 \mathrm{msec}$ after the vertical sync signal). This is because, in the raster scan displays (i.e., the raster scan CRT display and the LCD), the video signal takes about half of one frame duration to reach the center of the display - that is, the locus of the stimulus. On the other hand, the delay in onset was virtually absent in a vector scan display based on the CRT oscilloscope.

Stimuli. The target was a diamond-shaped figure subtending $0.51^{\circ}$ of visual angle with a missing corner $\left(0.08^{\circ}\right.$; top, bottom, left, or right); the missing corner varied randomly across trials. The mask was also diamond-shaped, but its larger frame could fit around the target. The mask subtended $1.00^{\circ}$ of visual angle. Both target and mask were green $\left(50 \mathrm{~cd} / \mathrm{m}^{2}\right.$ when they were displayed continuously), and both were presented in the center of a black background with a duration of $17 \mathrm{msec}$ each. Note that stimulus duration in LCDs simply represents the period of time from the onset of a stimulus to its offset, as determined, respectively, by switching on and off liquid crystal cells (sample-and-hold). On the other hand, in CRT displays, stimulus duration was defined by the number of pulses. Thus, LCDs 

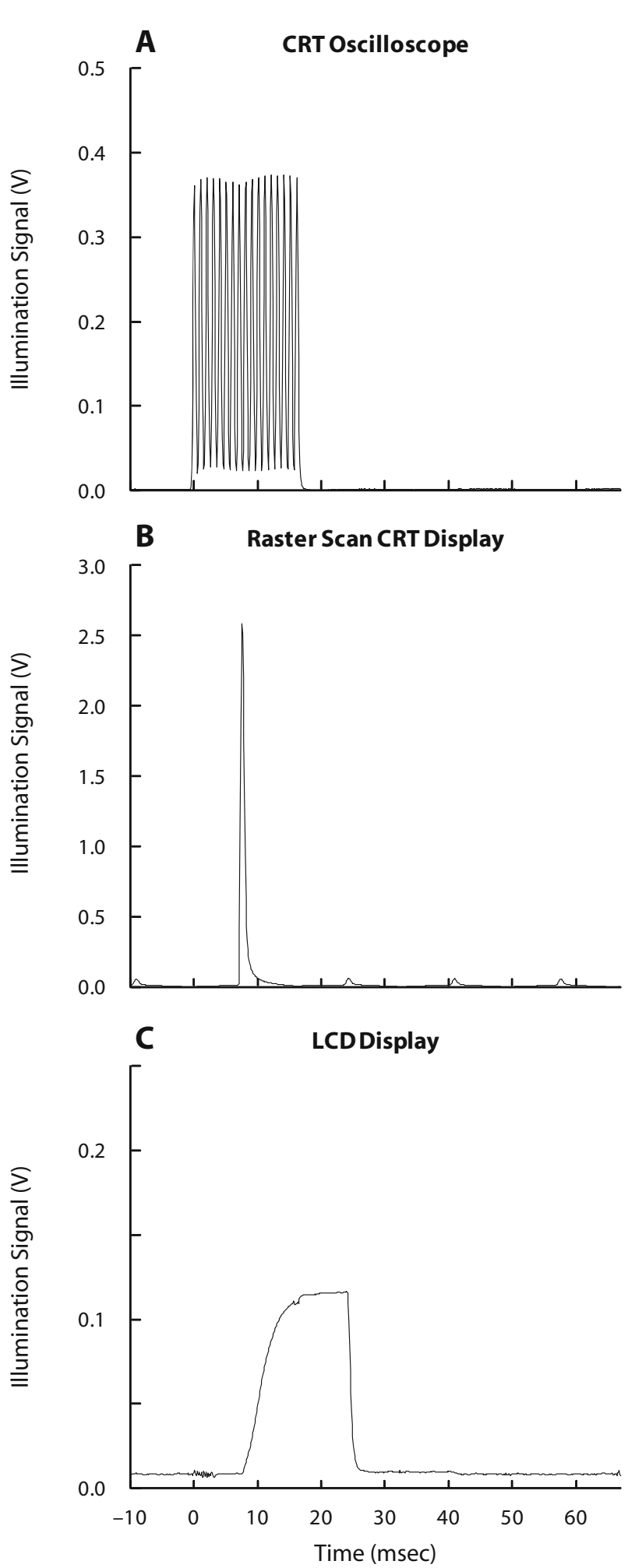

Figure 1. The average time course of the luminance changes for each monitor for a stimulus duration of $17 \mathrm{msec}$. (A) Luminance changes for the CRT oscilloscope. (B) Luminance changes for the raster scan CRT display. (C) Luminance changes for the LCD. The $y$-axis indicates the illumination signal (in volts, $V$ ) of the photodiode converted by the current-to-voltage conversion amplifier. The $x$-axis indicates the time (zero indicates the time when the computer sends a drawing signal to the display in the first frame). present the stimulus with approximately constant luminance during the presentation period, whereas the luminance varies substantially in CRT displays.

Design and Procedure. Two factors were manipulated within participants: monitor (CRT oscilloscope, raster scan CRT display, and LCD) and SOA between the target and mask $(0,17,33,50,67$, $83,100,117,133,167$, and $333 \mathrm{msec}$ and no-mask, in which the mask was not presented). Monitor condition was blocked, with each block administered twice. The order of blocks was counterbalanced across participants. Each block consisted of 240 trials ( 20 trials for each of the 12 SOA conditions) presented in a random order. A total of 1,440 trials were given to each participant ( 20 trials $\times 12$ SOA conditions $\times 3$ monitor conditions $\times 2$ blocks). Before an experimental session, participants performed 12 practice trials in the nomask condition. Experiment 1 took about $60 \mathrm{~min}$ to complete.

Figure 2 shows schematic illustrations of the event sequence for three different types of trials (conditions) in Experiment 1. All the trials began with a central fixation cross displayed for $500 \mathrm{msec}$, followed by the target. On trials with a 0 -msec SOA condition, the mask was simultaneously presented with the target. On no-mask trials, only the target was presented. On other trials, the mask was presented after the target at a particular SOA. Following the offset of a stimulus, participants had to identify the location of the missing corner of the target by pressing one of the arrow keys. There were no time constraints on the response, and participants responded at their own pace. They were encouraged to guess if they were uncertain about the missing corners. No feedback was provided.

\section{Results and Discussion}

Target accuracy is shown in Figure 3 as function of SOA and monitor type. Following previous metacontrastmasking studies (e.g., Breitmeyer et al., 2006), we conducted an ANOVA to evaluate differences between the experimental conditions. Accuracies were log transformed to adjust to a normal distribution of data assumed by ANOVA. The transformed data followed a normal distribution (Kolmogorov-Smirnov test, all $p \mathrm{~s}>.05$ ).

A two-way repeated measures ANOVA yielded significant main effects of SOA $[F(11,121)=16.32, p<.05$, $\left.\eta^{2}=.34\right]$ and monitor $\left[F(2,22)=8.02, p<.05, \eta^{2}<\right.$ $.01]$. In addition, the interaction between SOA and monitor was significant $\left[F(22,242)=2.08, p<.05, \eta^{2}=.01\right]$. Post hoc analyses (Tukey's HSD, $p<.05$ ) indicated that accuracy in the 33-msec SOA condition was lower than the accuracy levels for SOAs in the 0-, 83-, 100-, 117-, 133-, 167-, and 333-msec conditions and in the no-mask condition in all the monitor conditions. Furthermore, significant differences in accuracy between the raster scan CRT display and the CRT oscilloscope were observed in 50- and 67-msec SOA conditions; in addition, significant differences in accuracy were also observed between the raster scan CRT display and the LCD in the 33- and 50-msec SOA conditions.

Despite these quantitative differences, the results in all the monitor conditions showed the familiar U-shaped function characteristics of metacontrast masking. That is, the lowest accuracy in target identification was generally obtained in the 33-msec SOA condition. This implies that every monitor used in the present study exhibited fine enough temporal responses to investigate one of the main effects of metacontrast masking, even though the time courses of luminance changes are quite different between monitors. In the 50-msec SOA condition, the 
Fixation

(500 msec)

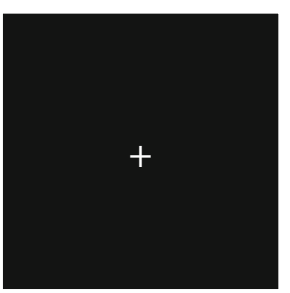

Fixation

(500 msec)

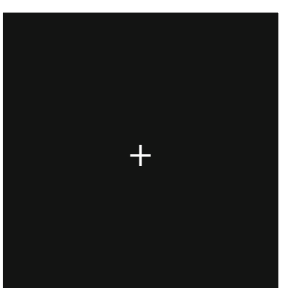

Fixation

(500 msec)

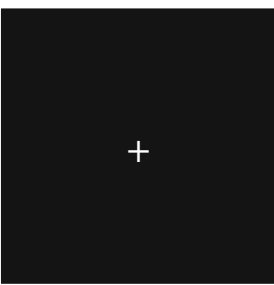

Target

(17 msec)

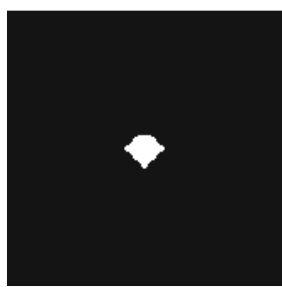

Target and Mask (17 msec)

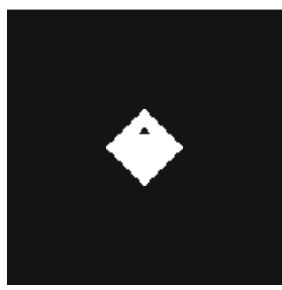

Target (17 msec)

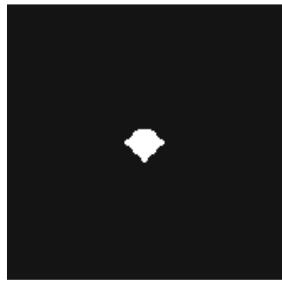

Blank (0-317 msec)

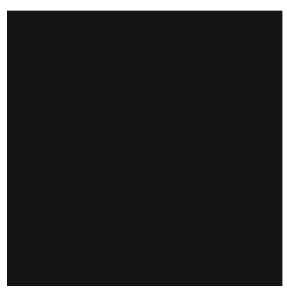

Blank (Until Response)

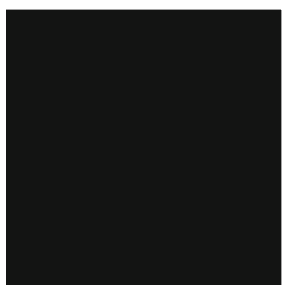

Blank (Until Response)

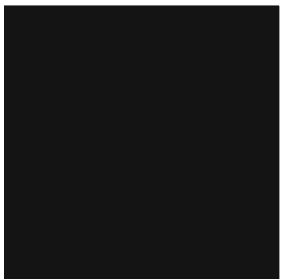

Blank

(17 msec)

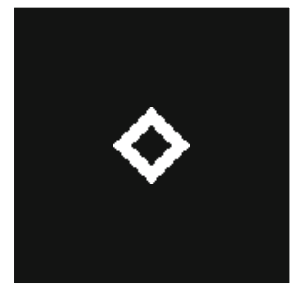

(Until Response)

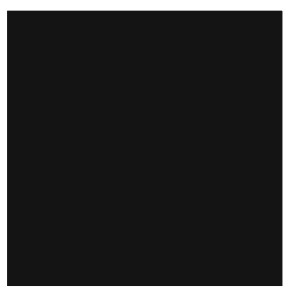

C

Figure 2. Schematic illustrations of the event sequence of different trial types in Experiment 1. (A) The conditions in which the stimulus onset asynchrony (SOA) between the target and the mask was $17 \mathrm{msec}$ or longer. (B) The condition in which the SOA was 0 msec. (C) The no-mask condition, in which the mask did not follow the target.

magnitude of the masking effect was significantly larger with the raster scan CRT display than with the other two monitors. This point is discussed in detail in the General Discussion section.

\section{EXPERIMENT 2}

The results of Experiment 1 indicated that a stable pattern of a metacontrast-masking effect could be obtained with LCDs. However, it remained unclear whether the similarities across monitors would be preserved for temporal attention. In Experiment 2, we examined whether differences among monitors have an impact on the attentional blink, a well-established phenomenon of temporal attention (Chun \& Potter, 1995; Shapiro et al., 1997).

\section{Method}

Participants. A new group of 12 adults ( 6 of them male and 6 female; age range, 19-22 years) from the participant pool of the Na- tional Institute of Advanced Industrial Science and Technology participated in this experiment. All the participants received payment for their participation. All had self-reported normal or corrected-tonormal vision.

Apparatus. The apparatus was the same as that used in Experiment 1 . Because the stimulus duration in Experiment 2 was $50 \mathrm{msec}$ (hence, it differed from that in Experiment 1), we measured the time course of the luminance changes produced by the onset of the filled green square presented for $50 \mathrm{msec}$, followed by a 50 -msec blank on each monitor $(\mathrm{SOA}=100 \mathrm{msec})$. Averages of 100 tests of the time course for luminance measurements, detailed earlier, are shown for each monitor type in Figure 4.

Stimuli. The RSVP stream consisted of 18 distractors and two targets. The distractors were randomly chosen digits (1-9). The same digit was not presented successively. Targets were randomly chosen uppercase alphabet letters, excluding I, O, Q, and Z. The first and the second targets were never the same. Each item was presented at the center of each monitor for $50 \mathrm{msec}$, with $50-\mathrm{msec}$ interstimulus intervals (presentation rate $=10$ items per second). The items, subtending $0.8^{\circ}$ vertically and $1.0^{\circ}$ horizontally in visual angle, were green $\left(50 \mathrm{~cd} / \mathrm{m}^{2}\right.$ when they were displayed continuously) against a black background. 


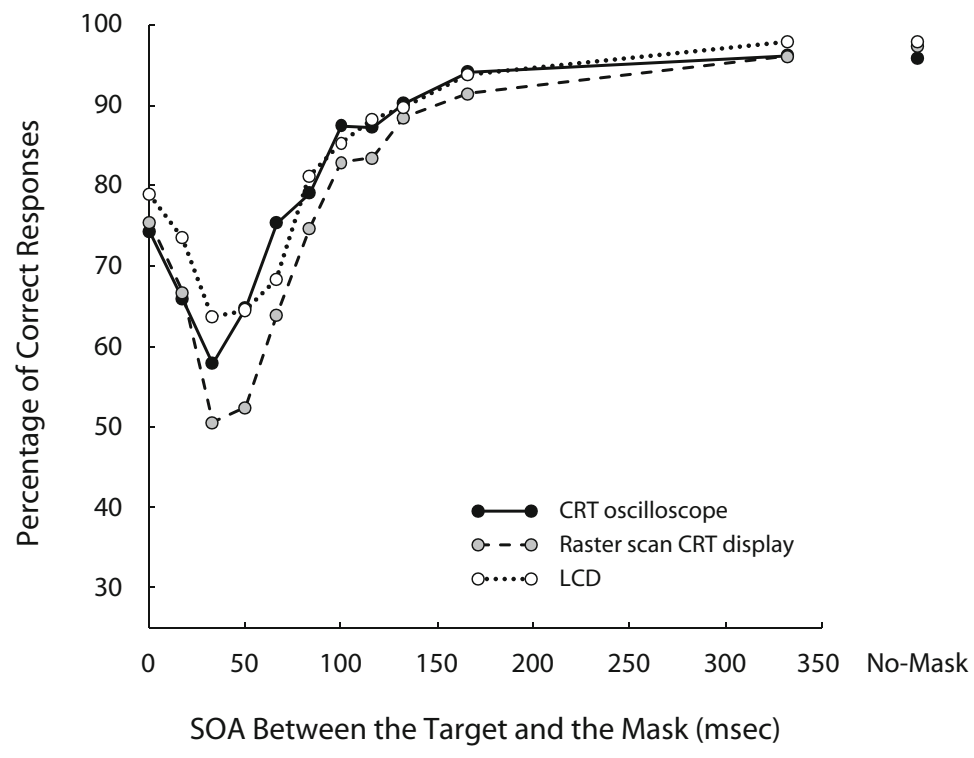

Figure 3. Mean correct identification response to the target as a function of stimulus onset asynchrony (SOA) between the target and the mask for each monitor in Experiment 1.

Design and Procedure. Two factors were manipulated within participants: monitor (CRT oscilloscope, raster scan CRT display, and LCD) and SOA between the first and second targets (100, 200, 300,500 , and $700 \mathrm{msec}$ ). Each monitor condition was blocked, and each block was administered twice. The order of blocks was counterbalanced across participants. Each block consisted of 100 trials (20 trials for each of the five SOA conditions) presented in random order. A total of 600 trials were given to each participant (20 trials $X$ 5 SOA conditions $\times 3$ monitor conditions $\times 2$ blocks). Before the experimental session, the participants performed 5 practice trials. Experiment 2 took about $60 \mathrm{~min}$ to complete.

Figure 5 illustrates the sequence of events on a trial in Experiment 2. Each trial began with a central fixation cross displayed until the space bar was pressed, followed by the RSVP stream. Within streams, the number of distractors before the first target varied between four and eight. The SOA between the first and second target was $100,200,300,500$, or $700 \mathrm{msec}$ (i.e., the second target was the first, second, third, fifth, or seventh item presented after the first target). After presentation of a stream, the participants identified two target letters by pressing two corresponding keys. There were no time constraints on the responses, and the participants responded at their own pace. They were encouraged to guess if they were uncertain about the identities of targets. No feedback about performance was provided.

\section{Results and Discussion}

Target identity responses were scored as correct regardless of report order. Following previous attentional blink studies (e.g., Raymond et al., 1992), we conducted ANOVAs to evaluate differences between experimental conditions. The log-transformed individual accuracy rates were submitted to a Kolmogorov-Smirnov normal distribution test (all $p \mathrm{~s}>.05$ ); then ANOVAs were performed.

Accuracy levels for the first target are shown in Figure $6 \mathrm{~A}$ as a function of SOA and monitor type. A two-way repeated measures ANOVA of correct responses resulted in neither a significant main effect of monitor $[F(2,22)=$ 0.08 , n.s., $\left.\eta^{2}<.01\right]$ nor a significant interaction of monitor with SOA $\left[F(8,88)=0.68\right.$, n.s., $\left.\eta^{2}<.01\right]$. However, there was a significant main effect of SOA $[F(4,44)=$ $\left.17.89, p<.05, \eta^{2}=.17\right]$. Post hoc analyses (Tukey's HSD, $p<.05)$ indicated that accuracy for the first target in the 100-msec SOA condition was lower than the accuracy levels in the other SOA conditions. Deterioration of identification accuracy, observed here for the first target in the 100-msec SOA condition, has also been reported elsewhere for SOAs between targets of $100 \mathrm{msec}$ or less (Chun \& Potter, 1995; Potter, Staub, \& O'Connor, 2002). This first target deficit, as well as the second target deficit (i.e., attentional blink), is assumed to reflect a late stage of visual processing that is modulated by temporal attention (Kawahara \& Enns, 2009; Potter et al., 2002). In Experiment 3 , we examined this first target deficit in more detail, using shorter SOAs between targets presented with different monitors.

Accuracy levels for the second target are shown in Figure $6 \mathrm{~B}$; these are based only on those trials on which the first target had been correctly identified. A two-way repeated measures ANOVA yielded a significant main effect of SOA $\left[F(4,44)=16.12, p<.05, \eta^{2}=.32\right]$. Post hoc analyses showed that accuracy levels for the second target were lower for SOAs of 200 and $300 \mathrm{msec}$ than they were for SOA conditions of 100,500 , and $700 \mathrm{msec}$; this indicates that the attentional blink effect occurred in Experiment 2 . There was also a significant main effect of monitor $\left[F(2,22)=4.42, p<.05, \eta^{2}<.01\right]$. Post hoc analyses showed that accuracy identifying the second target was lower with the LCD monitor than with either the CRT 

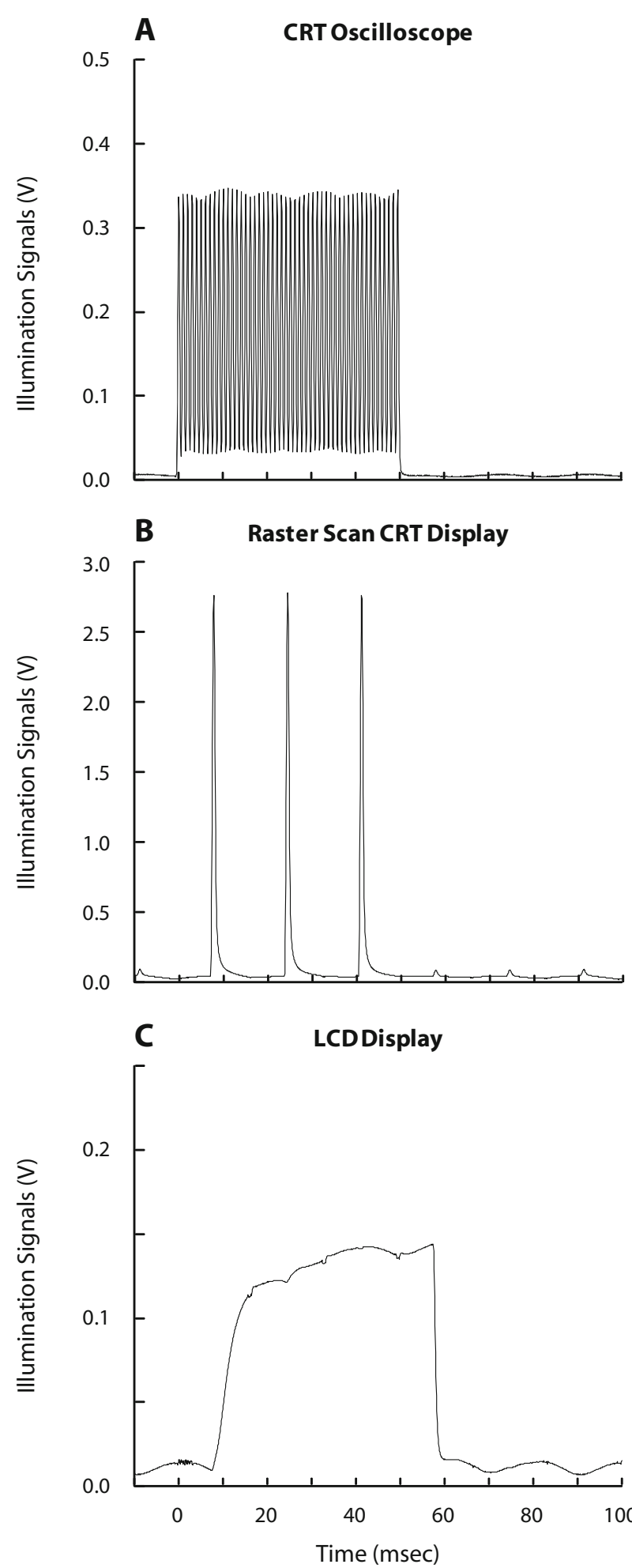

Figure 4. Average time course of the luminance change for each of three monitor types, with a stimulus duration of $50 \mathrm{msec}$. (A) Luminance changes for the CRT oscilloscope. (B) Luminance changes for the raster scan CRT display. (C) Luminance changes for the LCD. The $y$-axis indicates the illumination signal (in volts, $V$ ) of the photodiode converted by the current-to-voltage conversion amplifier. The $\boldsymbol{x}$-axis indicates the time (zero indicates the time when the computer sends a drawing signal to the display in the first frame). oscilloscope or the raster scan CRT display. The interaction of monitor with SOA was not statistically significant $\left[F(8,88)=1.04\right.$, n.s., $\left.\eta^{2}<.01\right]$.

Consistent with the findings in Experiment 1 involving metacontrast, these results showed that in all the monitor conditions a U-shaped function was found that is characteristic of the attentional blink. The lowest accuracy in identifying the second target occurred with the 200- and 300-msec SOA conditions (relative to the other SOA conditions) in all the monitor conditions. In other words, every monitor in the present study responded rapidly enough to permit examination of the attentional blink. Our results also showed that baseline accuracy for identifying the second target was significantly lower for the LCD than for the other two CRT monitors, although the interaction between monitor and SOA (i.e., the attentional blink effects across monitors) was statistically nonsignificant. The effect of the monitor may be due to the stimuli in the LCD having sharper edges than for the CRT monitors. These sharper edges may have led to stronger masking effects from the distractors.

\section{EXPERIMENT 3}

The results of Experiment 2 suggest that the attentional blink can be obtained with the LCD, as well as the CRT, monitors. However, it may be premature to conclude that the LCD has sufficient temporal precision to support investigations of the attentional blink, because it is known that the typical attentional blink profile over SOAs exceeding $100 \mathrm{msec}$ differs dramatically from profiles observed with SOAs of less than $100 \mathrm{msec}$. For example, Potter et al. (2002) reported that accuracy for identifications of a first target could be lower than accuracy levels for a second target that is presented within about $50 \mathrm{msec}$ following the first target. As target-to-target SOAs under $100 \mathrm{msec}$ increase, the accuracy of identifying the first target increases, and the accuracy of identifying the second target decreases (see also Bachmann \& Hommuk, 2005). This trade-off between the accuracy levels for first and second targets has been considered a reflection of a late stage of visual processing. For example, it may result from competition between the two targets for limited attentional resources (Kawahara \& Enns, 2009; Potter et al., 2002). In Experiment 3, we examined whether the LCD and CRT monitors would accurately document the tradeoff between the identification accuracies for the two targets in the attentional blink.

\section{Method}

A different group of 12 adults ( 10 of them male and 2 female; age range, 19-23 years) from the participant pool of the National Institute of Advanced Industrial Science and Technology participated in this experiment. All the participants received payment for their participation. All had self-reported normal or corrected-to-normal vision.

The apparatus and stimuli were the same as those in Experiment 2, except that each RSVP item was presented for $50 \mathrm{msec}$ with no interstimulus intervals (presentation rate $=20$ items per second). The design and procedure were the same as those in Experiment 2, except that the SOA factor was varied over five conditions: 50, 100, 150,250 , and $350 \mathrm{msec}$. 


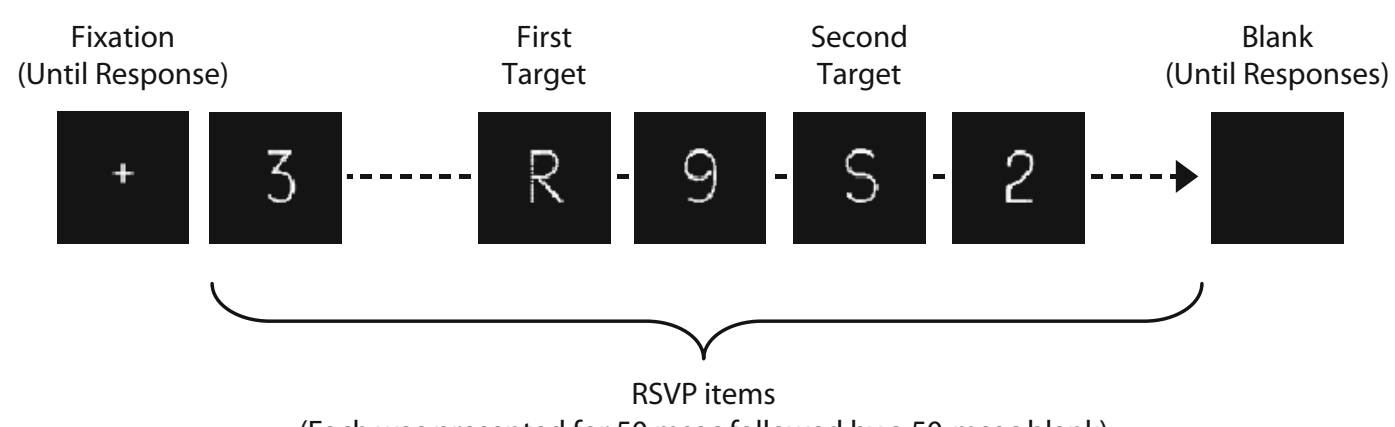

(Each was presented for 50 msec followed by a 50 -msec blank)

Figure 5. A schematic illustration of the event sequence in a trial in Experiment 2. The number of items between the targets was varied (zero, one, two, four, or six) according to the stimulus onset asynchrony (SOA) between the targets $(100,200,300,500$, or $700 \mathrm{msec})$.

\section{Results and Discussion}

Accuracy levels for first and second targets are shown in Figure 7 as a function of SOA and monitor. Following previous studies (e.g., Potter et al., 2002), ANOVAs were used to evaluate differences between the experimental conditions. The log transformed individual accuracy rates were submitted to a Kolmogorov-Smirnov normal distribution test (all $p \mathrm{~s}>.05$ ); then ANOVAs were performed.

A three-way ANOVA with target (first vs. second), SOAs (50-350 msec), and monitor (three types) as the main terms yielded a significant interaction between target and SOA $\left[F(4,44)=14.52, p<.05, \eta^{2}=.08\right]$. Post hoc analyses (Tukey's HSD, $p<.05$ ) indicated that accuracy in the 50-msec SOA condition for the first target was lower than that for the second target. In addition, the post hoc analyses showed that accuracies in the 250- and 350 -msec SOA conditions for the first target were higher than that for the second target. These results imply a tradeoff between the two target accuracies, and they also illustrate the localized presence of an attentional blink at longer SOAs (about $150 \mathrm{msec}$ ) (Kawahara \& Enns, 2009; Potter et al., 2002). Importantly, the ANOVA yielded no significant main effect or interactions including the factor of monitor: main effect of monitor $[F(2,22)=2.18$, n.s., $\eta^{2}=.01$; interaction between monitor and target $F(2,22)=1.81$, n.s., $\eta^{2}<.01$; interaction of monitor and SOA, $F(8,88)=1.20$, n.s., $\eta^{2}<.01$; interaction of monitor, target, and SOA, $F(8,88)=1.09$, n.s., $\left.\eta^{2}<.01\right]$. These results suggest that similar accuracy profiles over SOAs for first and second targets were obtained from the CRT oscilloscope, raster scan CRT display, and LCD.
A

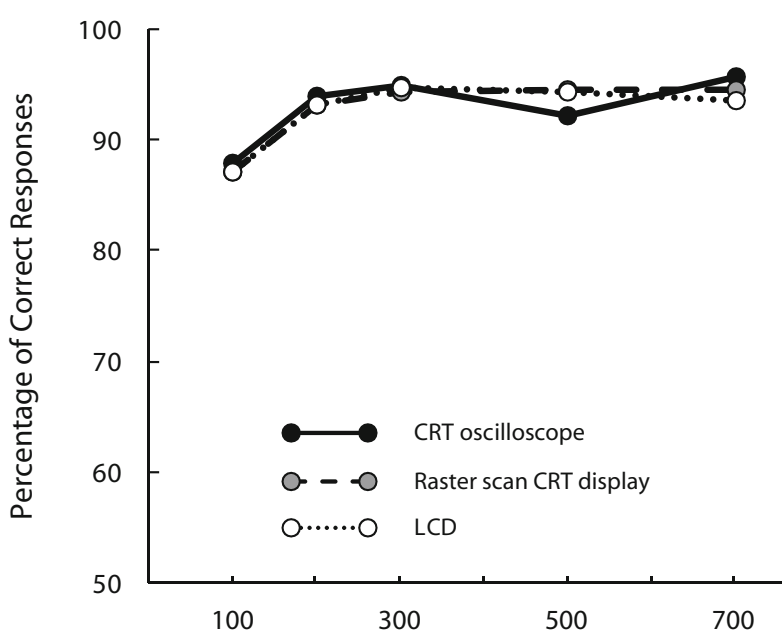

B

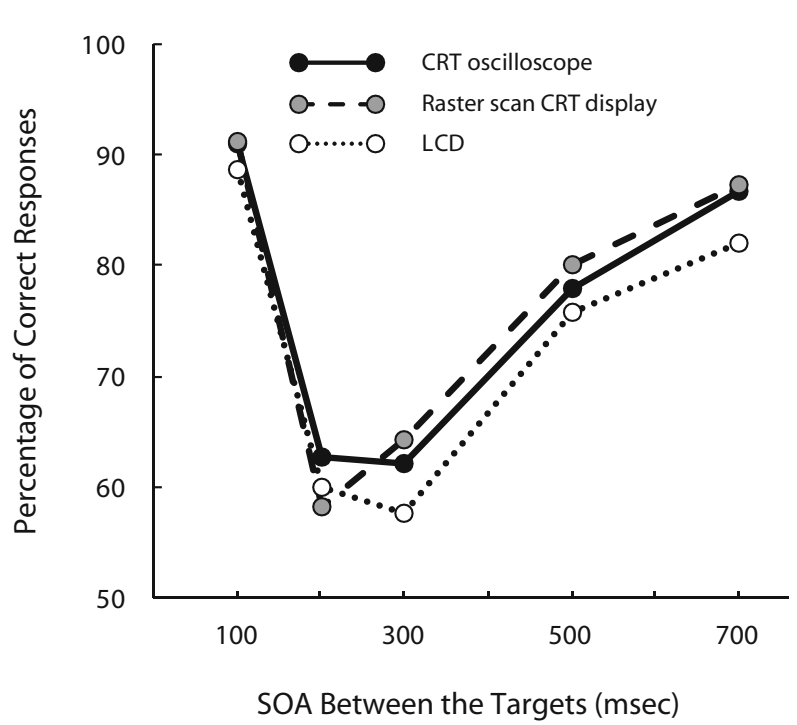

Figure 6. Results of Experiment 2. (A) Mean correct identification responses to the first target as a function of stimulus onset asynchrony (SOA) between the first and second targets. (B) Mean correct identification responses to the second target given correct report of the first target, as a function of SOA between the first and second targets. 

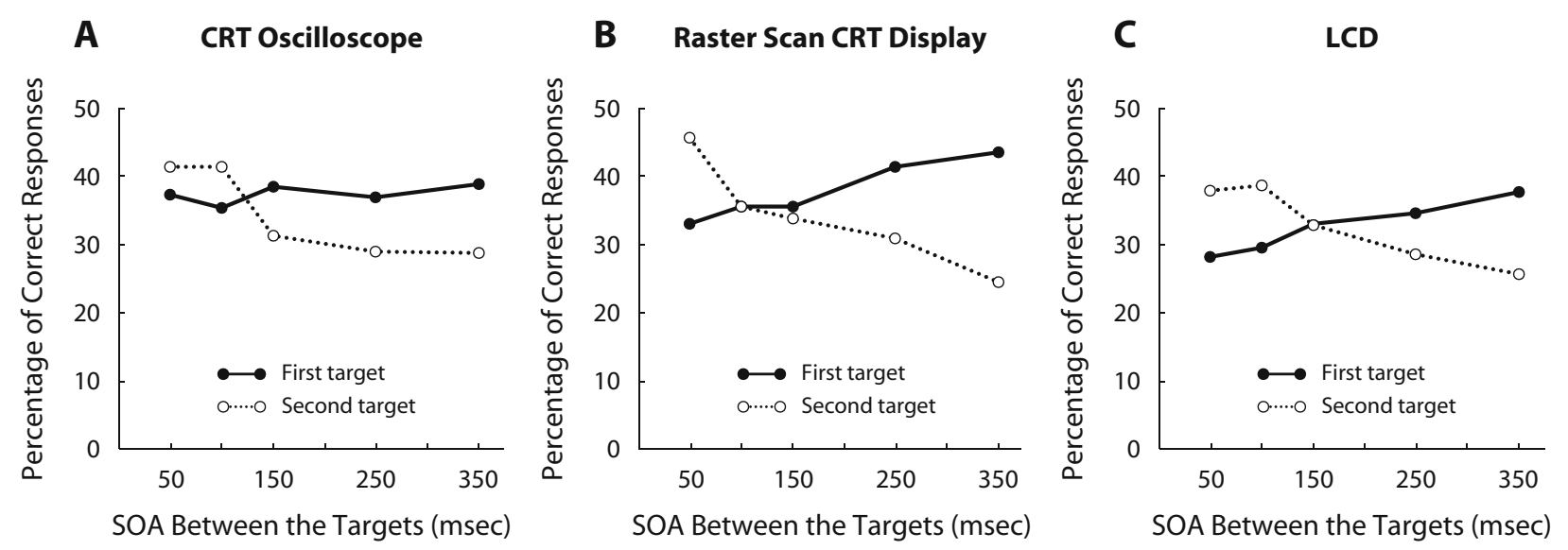

Figure 7. Results of Experiment 3. (A) Mean correct identifications of first and second targets as a function of stimulus onset asynchrony (SOA) between the targets in the condition with the CRT oscilloscope. (B) Mean correct identifications of first and second targets as a function of SOA between the targets in the condition with the raster scan CRT display. (C) Mean correct identifications of first and second targets as a function of SOA between the targets in the condition with the LCD.

\section{GENERAL DISCUSSION}

Traditionally, CRT oscilloscopes and raster scan CRT displays have been used as computer-controlled monitors in experimental studies investigating human vision. However, use of LCDs is becoming increasingly prevalent not only in our daily lives, but also in many fields of research. The purpose of the present study was to examine whether LCDs can yield typical behavioral profiles comparable to those of other displays used in visual tasks. To this end, performance in metacontrast-masking and attentional blink tasks was compared across three different display devices: an LCD, a CRT oscilloscope, and a raster scan CRT. Experiment 1 focused on the metacontrastmasking paradigm, which reflects early visual processing over time (Breitmeyer \& Öğmen, 2000). Experiments 2 and 3 focused on the attentional blink paradigm, which reflects a late stage of visual processing (Chun \& Potter, 1995; Kawahara \& Enns, 2009; Potter et al., 2002; Shapiro et al., 1997). Consistently, across all experiments, the results showed that all the monitors, including the LCD, provided patterns of target identification accuracies as a function of SOA that were typical of the performance profiles reported in previous studies. This indicates that the temporal operating characteristics of LCD used in the present study were sufficient to investigate metacontrast masking and the attentional blink, even though the time courses of luminance changes are quite different from those for CRT monitors.

In Experiment 1, the metacontrast-masking effect was greater in the raster scan CRT display, relative to the other two monitors. This result is possibly due to the fact that the instantaneous peak of luminance was clearly greater in the raster scan CRT display than in the other two monitors (see Figure 1), and this may affect the perceived intensity of visual stimuli. The metacontrast-masking effect is strongly influenced by mask intensity, as well as by the mask-target SOA (Breitmeyer \& Öğmen, 2000). That is, the metacontrast-masking effect increases with increases in the mask intensity. It is possible that the higher peak of luminance in the raster scan CRT display increased mask intensity, relative to the other two monitors, thereby resulting in the greater metacontrast-masking effect. Another possibility is that clarity of the stimuli was related to a stronger masking effect in the raster scan CRT display, because the CRT display produced blurry images, relative to the LCD display (Krupinski et al., 2004), resulting in a greater effect of contour proximity (Alpern, 1953) under the critical masking period.

The results revealed that the LCD used in the present study provided temporal operating characteristics sufficient to obtain a typical metacontrast-masking and attentional blink effect. However, this does not guarantee that all types of LCDs will yield similar results. We do not argue that any LCDs and CRTs are equivalent; it is not certain whether the same pattern of results will be obtained with LCD and CRT displays for different types of target and masking stimuli. Furthermore, even with the use of the same LCD as that in the present study, it remains unclear whether the display can provide appropriate timing controls in other experimental paradigms. In the present study, we did not assess the physical properties of the monitors during experimental trials. The controllability of the physical properties of a monitor over trials is also an important factor in psychological research.

Of course, LCD monitors may not be appropriate for some investigations, but the results here suggest that at least some robust empirical findings can be produced with a wide variety of monitors. It is unclear whether one monitor is fundamentally better than another with regard to these empirical findings. Although it is important for researchers to know the details of their monitors, it is comforting to know that at least some important empirical findings are robust enough that the differences across monitors do not matter very much. It will be important to provide continuous assessments of the temporal operating characteristics of LCDs (and related devices) for the research community in order for researchers to 
make effective use for a broad range of experimental paradigms.

\section{AUTHOR NOTE}

This work was partially supported by grants from the Japan Society for the Promotion of Science to K.K. and J.-I.K. We thank Gregory Francis and anonymous reviewers for very helpful comments on a draft of this article. Correspondence concerning this article should be addressed to K. Kihara, National Institute of Advanced Industrial Science and Technology, Central 6, 1-1-1 Higashi, Tsukuba 305-8566, Japan (e-mail: ken-kihara@aist.go.jp).

\section{REFERENCES}

Alpern, M. (1953). Metacontrast. Journal of the Optical Society of America, 43, 648-657.

Bach, M., Meigen, T., \& Strasburger, H. (1997). Raster-scan cathoderay tubes for vision research - Limits of resolution in space, time and intensity, and some solutions. Spatial Vision, 10, 403-414.

BachmanN, T., \& HommuK, K. (2005). How backward masking becomes attentional blink: Perception of successive in-stream targets. Psychological Science, 16, 740-742.

BECKER, M. E. (2008). LCD response time evaluation in the presence of backlight modulations. SID Symposium Digest, 39, 24-27.

Brainard, D. H. (1997). The Psychophysics Toolbox. Spatial Vision, 10, 433-436.

Breitmeyer, B. G., Kafaligonul, H., Öğmen, H., Mardon, L., Todd, S., \& Ziegler, R. (2006). Meta- and paracontrast reveal differences between contour- and brightness-processing mechanisms. Vision Research, 46, 2645-2658.

BreitMeyer, B. G., \& ÖĞMEN, H. (2000). Recent models and findings in visual backward masking: A comparison, review, and update. Perception \& Psychophysics, 62, 1572-1595.

BreitMeyer, B. G., \& ÖĞMEN, H. (2006). Visual masking: Time slices through conscious and unconscious vision (2nd ed.). New York: Oxford University Press.

Chun, M. M., \& PotTer, M. C. (1995). A two-stage model for multiple target detection in rapid serial visual presentation. Journal of Experimental Psychology: Human Perception \& Performance, 21, 109-127.

Di Lollo, V., Seiffert, A. E., Burchett, G., Rabeeh, R., \& Ruman, T. A. (1997). Phosphor persistence of oscilloscopic displays: A comparison of four phosphors. Spatial Vision, 10, 353-360.
Elze, T., \& TAnner, T. G. (2009). Liquid crystal display response time estimation for medical applications. Medical Physics, 36, 49844990.

KaWahara, J., \& EnNS, J. T. (2009). Selection difficulty and interitem competition are independent factors in rapid visual stream perception. Journal of Experimental Psychology: Human Perception \& Performance, 35, 146-158.

Krupinski, E. A., Johnson, J., Roehrig, H., Nafziger, J., Fan, J., \& LUBIN, J. (2004). Use of a human visual system model to predict observer performance with CRT vs. LCD display of images. Journal of Digital Imaging, 17, 258-263.

LiANG, H., \& BADANO, A. (2007). Temporal response of medical liquid crystal displays. Medical Physics, 34, 639-646.

Menozzi, M., NÄPflin, U., \& KRUeger, H. (1999). CRT versus LCD: A pilot study on visual performance and suitability of two display technologies for use in office work. Displays, 20, 3-10.

Pelli, D. G. (1997). The VideoToolbox software for visual psychophysics: Transforming numbers into movies. Spatial Vision, 10, 437442.

Potter, M. C., Staub, A., \& O'Connor, D. H. (2002). The time course of competition for attention: Attention is initially labile. Journal of Experimental Psychology: Human Perception \& Performance, 28, 1149-1162.

Raymond, J. E., Shapiro, K. L., \& Arnell, K. M. (1992). Temporary suppression of visual processing in an RSVP task: An attentional blink? Journal of Experimental Psychology: Human Perception \& Performance, 18, 849-860.

Shapiro, K. L., Arnell, K. M., \& Raymond, J. E. (1997). The attentional blink. Trends in Cognitive Sciences, 1, 291-296.

Tourancheau, S., Callet, P. L., \& Barba, D. (2008). Image and video quality assessment using LCD: Comparisons with CRT conditions. IEICE Transactions on Fundamentals of Electronics, Communications \& Computer Sciences, E91-A, 1383-1391.

Watson, A. B., Nielsen, K. R. K., Poirson, A., Fitzhugh, A., Bilson, A., Nguyen, K., \& AhumadA, A. J., JR. (1986). Use of a raster framebuffer in vision research. Behavior Research Methods, Instruments, \& Computers, 18, 587-594.

Wiens, S., Fransson, P., Dietrich, T., Lohmann, P., Ingvar, M., \& Öhman, A. (2004). Keeping it short: A comparison of methods for brief picture presentation. Psychological Science, 15, 282-285.

(Manuscript received October 29, 2009; revision accepted for publication April 3, 2010.) 